\title{
Aproximación a la validación de un juguete inteligente como apoyo en la detección precoz de problemas del desarrollo
}

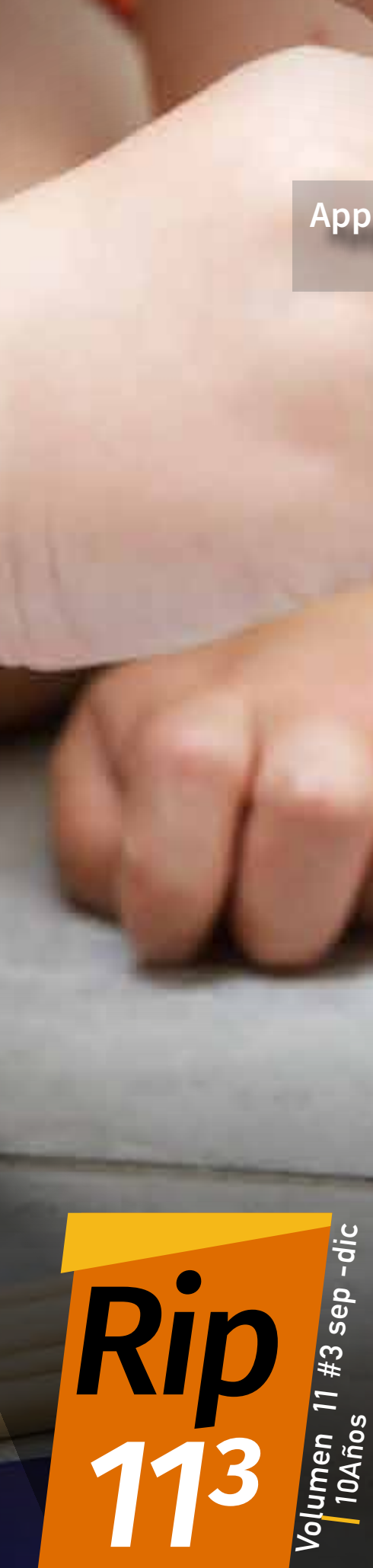

Approach to the validation of an intelligent toy as a support tool in the early diagnosis of developmental disorders

(c) $($ i) $(9)$

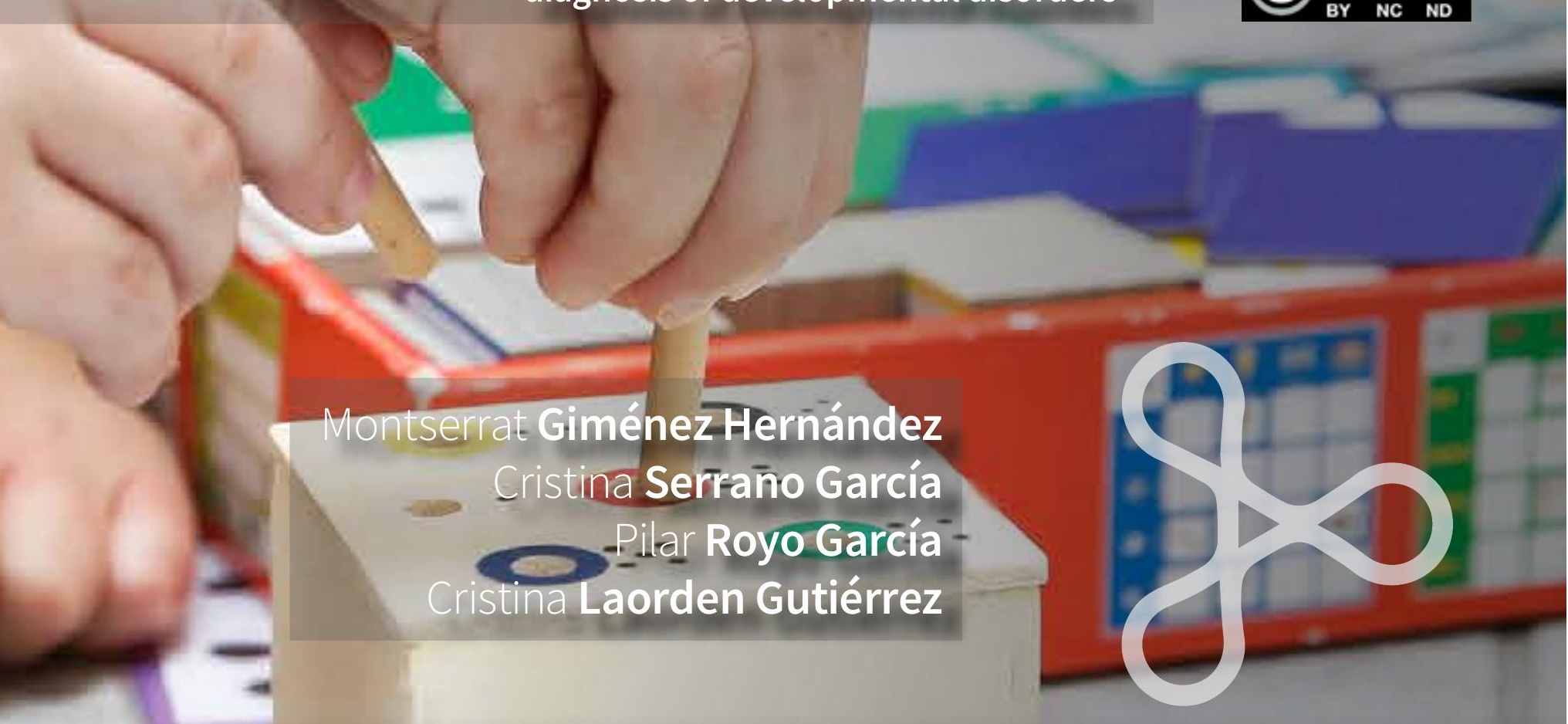


ISSN-I: 2027-1786 | e-ISSN: 2500-6517 Publicación Cuatrimestral
ID:

\section{7-1786.RIP.11305}

Title: Approach to the validation of an intelligent toy as a support tool in the early diagnosis of developmental disorders

Título: Aproximación a la validación de un juguete inteligente como apoyo en la detección precoz de problemas del desarrollo

Alt Title / Título alternativo:

[es]: Aproximación a la validación de un juguete inteligente como apoyo en la detección precoz de problemas del desarrollo

Author (s) / Autor (es):

Giménez Hernández, Serrano García , Royo García, \& Laorden Gutiérrez

Keywords / Palabras Clave:

[es]: Psicologia del Desarrollo, Tecnología

Proyecto / Project:

Proyecto de I+D+i "Ecosistema de Detección Ubicua, atenCión y Estimulación tempRana para niños con trastornos del dEsarrollo (EDUCERE)" abordado por un equipo transdisciplinar integrado por investigadores de la Universidad Politécnica de Madrid, la Unive

Financiación / Funding:

Proyecto de I+D+i “Ecosistema de Detección Ubicua, atenCión y Estimulación dEsarrollo (EDUCERE)" abordado por un equipo transdisciplinar integrado por investigadores de la Universidad Politécnica de Madrid, la Unive

Submited: tempRana para niños con trastornos del 2018-04-16
Resumen

Este artículo presenta un acercamiento a la validación de un juguete inteligente (desarrollado en el marco del proyecto

EDUCERE), que aproxime a la detección no invasiva de trastornos de desarrollo del niño. El juguete consiste en una torre de 5 cubos en los que se han insertado sensores para medir una serie de parámetros. Para la recogida de datos,

se contó con una muestra de 68 niños entre

24 y 48 meses. Además, a una submuestra de 27 niños se aplicó la escala de desarrollo

Merrill Palmer-R. Los resultados parecen mostrar que el $n^{\circ}$ de cubos utilizados en la construcción de la torre, el $n^{\circ}$ de movimientos que realizan y la velocidad y aceleración de los movimientos son las variables más relevantes

en la elaboración de la torre. El nivel de ejecución de la torre, evaluada a través de los sensores, se relaciona con diversas áreas del desarrollo infantil; siendo la motricidad gruesa el área de desarrollo que más diferencia entre

los niños que construyeron bien la torre y los que la hicieron mal. Estos resultados apoyan la adecuación de los cubos inteligentes y sirven

para futuras investigaciones que validen de forma consistente su utilidad para la detección de problemas de desarrollo.

\section{Abstract} a smart toy (developed within the framework of a larger project called EDUCERE), with the aim of detecting developmental disorders in children, in an early, non-invasive manner. The toy consists of a tower of 5 cubes in which

sensors have been inserted to measure a series of parameters. The sample was made up of 68 children between the ages of 24 and

48 months. The Merrill Palmer-R Scales of Development were applied to a sub-sample of 27 children. The results seem to show that the number of cubes used by the children in the construction of a tower, the number of movements they perform, as well as the speed and acceleration of their movements, are the most relevant variables in the construction of the tower. The execution level in the task of building of the tower - measured by the sensors- is related to various areas of child development, such as gross and fine motor skills, social emotional and cognitive aspects, and the general intelligence level. Of these, gross motor skills are the area of development that differentiates more the children who built the tower correctly from those who did not. These results support the use of the smart cubes and serve as a precedent for future researches that aim to validate consistently their use in the detection of developmental problems.
This article is an approach to the validation of

\section{Citar como:}

Giménez Hernández, M., Serrano García, C. ., Royo García, P., \& Laorden Gutiérrez, C. . (2018). Aproximación a la validación de un juguete inteligente como apoyo en la detección precoz de problemas del desarrollo. Revista Iberoamericana de Psicología issn-l:2027-1786, 11 (3), 59-70. Obtenido de: https://revistas. iberoamericana.edu.co/index.php/ripsicologia/article/view/1413-4528
Dra Montserrat Giménez Hernández, Psi

Research ID: F-1155-2017

ORCID: https://orcid.org/0000-0001-8810-469

Source | Filiacion:

Universidad de Alcalà

$\mathrm{BIO}$

Doctora en Psicología. En la actualidad, Directora Gestora del Centro Universitario Cardenal Cisneros, adscrito a la Universidad de Alcalá. Docente Pregrado en Psicología y en el Máster Oficial de Atención a la Diversidad y Apoyos Educativos.

City | Ciudad:

Alcalá de Henares [es]

e-mail:

montse.gimenez@cardenalcisneros.es
Dra Cristina Serrano García , Psi

Research ID: E-8418-217

ORCID: https://orcid.org/0000-0002-6577-2007

Source | Filiacion:

Universidad de Alcalá

BIO:

Doctora en Psicopedagogía, Diplomada en Magisterios de Audición y Lenguaje. Acreditada po la ACAP para las cuatro figuras docentes. Profesora titular del Centro Universitario Cardenal Cisneros. Directora del Máster Oficial en Atención a la Diversidad y Apoyos Educativos en el Centro Universitario Cardenal Cisneros.

City | Ciudad:

Alcalá de Henares [es]

e-mail:

cristina.serrano@cardenalcisneros.es
Dra Pilar Royo García, Psi

Research ID: E-8913-2017

ORCID: https://orcid.org/0000-0003-0895-9286

Source | Filiacion:

Universidad de Alcalá

BIO:

Doctora en Psicología. Acreditada por la ACAP. Profesora titular del Centro Universitario Cardenal Cisneros en Pregrado y Maestría. Durante nueve años fue miembro del equipo investigador de la Unidad de Psicología Preventiva del Departamento de Psicología Evolutiva y de la Educación de la Universidad Complutense.

City | Ciudad:

Alcalá de Henares [es]

e-mail:

pilar.royo@cardenalcisneros.es
Dra Cristina Laorden Gutiérrez, Psi

Research ID: E-8850-2017

ORCID: https://orcid.org/0000-0001-7770-4234

Source | Filiacion:

Universidad de Alcalá

$\mathrm{BIO}$

Doctora en CC. De la Educación. Profesora titular en el Centro Universitario Cardenal Cisneros, adscrito a la Universidad de Alcalá, donde imparte docencia en los grados de Magisterio y de Educación Social. Subdirectora de Ordenacion Educación Social. Subdrectora de Ordenación Académica en el CU Cardenal Cisneros.

City | Ciudad:

Alcalá de Henares [es]

e-ma

cristina.laorden@cardenalcisneros.es 


\section{Aproximación a la validación de un juguete inteligente como apoyo en la detección precoz de problemas del desarrollo \\ Montserrat Giménez Hernández Cristina Serrano García Pilar Royo García Cristina Laorden Gutiérrez}

\section{Introducción}

La Organización Mundial de la Salud (OMS) indicó en su informe "World report on disability" (Alvarez, Soria, \& Sanchez, 2009), publicado en 2011, que para ese entonces aún había muchos casos de niños con trastornos del desarrollo que no eran correctamente identificados, ni recibían mecanismos de acción por parte de los servicios sanitarios (World Health Organization, 2011). En la actualidad, todavía hay mucho por hacer en el campo del diagnóstico precoz, en la detección temprana (Alcantud, Alonso, \& Rico, 2015; Canal \& Fuentes, 2006), aún resulta difícil precisar la mejor manera de identificar a los/as niños/as con problemas del desarrollo (Alvarez, Soria, \& Sanchez, 2009). Los instrumentos más utilizados actualmente para evaluar el desarrollo del niño/a (cuya revisión es posible encontrar, por ejemplo, en García Sánchez, Mendieta, \& Castellanos (2010) resultan esenciales para la detección precoz de problemas y alteraciones, sin embargo, no todos los niños quienes lo necesitan tienen acceso a éstos (o a una evaluación que los emplee) al no haber una alerta precoz que conduzca a dicha evaluación. Por esta razón es fundamental poner en marcha, cuanto antes, procedimientos que permitan recoger información sobre las capacidades o dificultades del niño/a, en entornos naturales y ecológicamente relevantes para él, y que formen parte de sus rutinas habituales (Valero, 2001), lo anterior como paso previo a la remisión a profesionales.

Siguiendo esta línea, el juguete se convierte en una herramienta privilegiada dada su contribución bien sabida -y la del juego en generalal desarrollo global del ser humano (Barrera, Perdomo, Serrato, \& Trujillo, 2014). Los juguetes son considerados instrumentos esenciales durante la infancia por su carácter altamente motivante y por generar aprendizaje y emociones que contribuyen a la socialización y a la construcción de la propia identidad. Así pues, mediante el juego el/ la niño/a favorece su desarrollo, su integración social, potencia su autoestima y desarrolla autonomía personal (Costa, y otros, 2007).

Considerando lo anterior, se cree que incorporar diferentes tipos de sensores en los juguetes que los niños usan cotidianamente permitirá recoger información sistemática sobre procesos y acciones, lo cual facilita, además, una detección precoz de posibles problemas del desarrollo que puedan afectar, en especial, la movilidad. Además, una vez detectados dichos problemas, será posible hacer uso de esta tecnología para atenderlos de manera temprana, mediante actividades educativas que puedan paliar, en lo posible, los efectos que estos problemas puedan generar en el futuro (Serrano \& Laorden, 2015).

El uso de juguetes cotidianos y familiares para los/as niños/as, que puedan servir como herramientas facilitadoras del diagnóstico temprano en situaciones naturales y cotidianas, está basado en tres pilares: internet de las cosas, el diseño para todos y el diseño universal. El concepto Internet de las Cosas refiere a la interconexión de objetos cotidianos con internet, a través de dispositivos como sensores y actuadores (Ashton, 2009; De Miguel, 2001). La filosofía o modelo diseño para todos (Rodríguez-Porrero \& Gil González, 2015) tiene como fin diseñar objetos, servicios, entornos, entre otros, teniendo en cuenta la diversidad humana, la inclusión social y la igualdad, de tal modo que puedan ser utilizados por el mayor número de personas posible. Por último, el paradigma del diseño universal (Rodríguez-Porrero \& Gil González, 2015) apunta al desarrollo de productos, entornos, programas y servicios que puedan ser utilizados por la mayor cantidad posible de personas, sin necesidad de adaptación ni diseño especializado, por lo que está anclado al concepto de accesibilidad. Resulta preciso 
agregar que los requisitos de las nuevas tecnologías para la interacción accesible son un aspecto fundamental para la integración e inclusión de niños/as. Dichos requisitos están detallados en la conferencia de clausura de TecnoNEEt 2010 - 25 años de Integración en España, en la que fueron contempladas las claves necesarias para la interacción del niño con "objetos de aprendizaje", útiles en los procesos de detección precoz y atención temprana (Valero, 2001). CEAPAT publicó hace unos años un documento titulado "Diseño para todos en educación" en el que fueron recopiladas las normativas actuales, opiniones de expertos e iniciativas existentes en favor del diseño para todos en educación (Rodríguez-Porrero \& Gil González, 2015).

En los últimos años han surgido estudios que emplean la tecnología para evaluar el movimiento de niños/as con algún tipo de dificultad, siendo uno de ellos el de Albinali, Goodwin, \& Intille (2012), en el que se presentan los datos recogidos por diferentes tipos de sensores para detectar estereotipo de aleteo de manos y balanceo corporal en individuos con TEA. Sin embargo, es preciso anotar que los sensores fueron colocados en ambas muñecas y el torso del niño/a, por lo cual es un procedimiento invasivo y presenta una situación poco habitual para los/as niños/as. En otro estudio, Smits-Engelsman, Wilson, Westenberg, \& Duysens (2003) registraron los movimientos de 32 niños/as con problemas de coordinación y aprendizaje, haciendo uso de un lápiz electrónico para dibujar sobre una tableta digital. El sistema permitía registrar el movimiento realizado por los niños, recogiendo valores de parámetros como la velocidad, la longitud del dibujo, la presión, etc. Tras analizar los resultados encontraron que, en comparación con un grupo control, los niños/as presentaban problemas de aprendizaje motor; a pesar que no hubo diferencias en los tiempo de respuesta (incluso, en ocasiones, los niños quienes presentaban problemas eran más rápidos), sí hubo diferencias en la velocidad de ejecución respecto al grupo control, en especial cuando los/as niños/as tenían problemas para controlar el movimiento (por ejemplo, cuando hacían un dibujo circular frente a uno lineal).

El interés de estos y otros estudios en evaluar aspectos motores no es gratuito. Si bien Manga \& Ramos (2001), establecen que las áreas indispensables para la evaluación y detección precoz de trastornos o dificultades del desarrollo son: percepción; lenguaje; memoria; cognición general; y motricidad, ésta última resulta especialmente fundamental en la evaluación de niños/as en edades más tempranas del desarrollo. Lo anterior en tanto los aspectos motores son buenos indicadores del nivel de maduración del sistema nervioso, son fácilmente mensurables y, en la evaluación de niños/as más pequeños, son una medida más sensible en comparación con las medidas cognitivas (Roid \& Sampers, 2011). Para lograr consistencia interna en cualquier evaluación es necesario apoyarse en alguno de los instrumentos desarrollados para la valoración del desarrollo psicomotor en edades tempranas (Robaina, Riesgo, \& Robaina, 2007; Cools, Martelaer, Samaey, \& C., 2008). Además, los aspectos motores son consideradas predictores significativos de problemas del desarrollo (Alcantud, Alonso, \& Rico, 2015; Roid \& Sampers, 2011; Iceta \& Yoldi, 2002) y existe evidencia de la fuerte relación entre desarrollo motor y otras patologías (Brown, 2000; Ramus \& Pidgeon, 2003; Tseng \& Yao, 2004; Diario de Alcala). Por último, la evaluación del desarrollo motor, en sí misma, puede servir para favorecer la competencia motriz de los escolares (Ruiz, Linaza, \& Peñaloza, 2008).

La presente investigación está enmarcada dentro de un proyecto más amplio (Proyecto EDUCERE) cuyo objetivo es investigar, desarrollar y evaluar soluciones innovadoras que permitan detectar alteraciones en el desarrollo psicomotor de los niños/as, a la vez que se llevan a cabo actividades de estimulación y de atención temprana. Con miras a lograr dicho objetivo, el proyecto EDUCERE propone el modelo "internet de los juguetes" (IoT, por sus siglas en inglés), basándose en el paradigma "internet de las cosas" (IoT) (Ashton, 2009), para desarrollar juguetes inteligentes basados en objetos cotidianos, que no sólo faciliten la interacción de los niños/as con éstos en entornos como la casa y la escuela, sino que les permitan recabar la información de manera no intrusiva (Nuñez-Nagy, Alarcos, Valero, Ramírez, \& Serrano, 2015; Martin-Ruiz, Valero, Linden, Nunez-Nagy, \& Gutierrez, 2015; Rivera, y otros, 2016) Martínez-Yelmo, 2016). Gracias a ésta información, será posible detectar trastornos del desarrollo en niños/as en el momento en que se presenten (Martin-Ruiz, Valero, Linden, Nunez-Nagy, \& Gutierrez, 2015; Rivera, y otros, 2016).

"Juguete inteligente" es considerado aquel quetiene componentes electrónicos (uno o más microprocesadores o microcontroladores; memoria; dispositivos de almacenamiento; y dispositivos de entrada y salida (Boss, Bruce, Charlie, \& Miller, 2001) y puede conectarse por red a otros juguetes inteligentes o a un ordenador (Shwe, 1999; Jelinek \& McGowan, 2001). En general, aquel que es controlado por software (Paula, 2008).

El juguete inteligente desarrollado en el seno del Proyecto EDUCERE (Nuñez-Nagy, Alarcos, Valero, Ramírez, \& Serrano, 2015; Martin-Ruiz, Valero, Linden, Nunez-Nagy, \& Gutierrez, 2015; Rivera, y otros, 2016), y empleado en la presente investigación, es una torre de 5 cubos, cada uno con sensores y otros dispositivos previamente insertados que permiten medir y almacenar una serie de parámetros. Teniendo en cuenta que la torre de cubos se utiliza en la mayoría de pruebas de inteligencia y de desarrollo infantil (por ejemplo, Escalas McCarthy; test de Denver; Haizea-Llevant, Test Brunet-Lezine; MerrillPalmer; etc.), y que construir una torre de cubos presenta ventajas para los niños (resumidas en Martin-Ruiz, Valero, Linden, Nunez-Nagy, \& Gutierrez (2015), en el proyecto EDUCERE se tomó la decisión de comenzar empleando éste juguete. En cuanto al desarrollo de los niños/as -en relación con la construcción de torres de cubos- se sabe que a los 18 meses los niños/as construyen torres de 2 cubos, a los 24 meses construyen torres de 6 cubos y a los 36 meses construyen torres de más de 6 cubos (Alcantud, Alonso, \& Rico, 2015). A medida que aumenta la complejidad, resulta necesario un mayor desarrollo de todas las habilidades implicadas en ésta tarea: coordinación manoojo; compresión de la relación entre dos objetos en el espacio; destreza manual; y capacidad de imitar. En general, la construcción de torres representa la habilidad del niño/a para manipular y ordenar objetos en diferentes configuraciones espaciales.

Recogiendo todo lo mencionado hasta el momento, éste artículo presenta un primer acercamiento a la validación de un juguete inteligente, a saber, la torre de cubos, como instrumento que ayude en la detección precoz de trastornos del desarrollo de niños/as, de manera no invasiva y en sus contextos cotidianos. Lo anterior, con miras a que la remisión a un profesional pueda ser llevada a cabo lo antes posible

El objetivo principal es analizar la correspondencia entre los datos recogidos por los sensores de los cubos inteligentes, el desempeño de los/as niños/as en la construcción de la torre y los resultados de la evaluación del desarrollo psicológico (tras la aplicación de la escala Merrill-Palmer-R). Dicho análisis permitirá conocer qué información aportada por los cubos resulta más relevante a la hora de detectar problemas del desarrollo en niños/as. 


\section{Método}

\section{Muestra}

La muestra la conformaron 68 sujetos (33 niños y 35 niñas) con edades entre los 24 y los 48 meses, pertenecientes a familias de clase media, y matriculados en tres (3) escuelas infantiles situadas en la Comunidad de Madrid (España).

Adicionalmente, una submuestra de 27 niños/as (12 niñas y 15 niños) fue conformada para explorar la posible relación entre los datos obtenidos por los sensores en los cubos y los resultados de la evaluación del desarrollo infantil (tras la aplicación de la escala Merrill Palmer-R) se obtuvo una submuestra que consta de 27 niños: 12 niñas y 15 niños

\section{Procedimiento}

Para poder realizar la actividad de la torre de cubos con los niños/as (Figura 1) y recoger datos, fue solicitada la autorización de las familias y de la dirección de los tres centros. Además, la educadoras correspondiente de los niños/as estuvo siempre presente y todo el proceso fue grabado en vídeo.

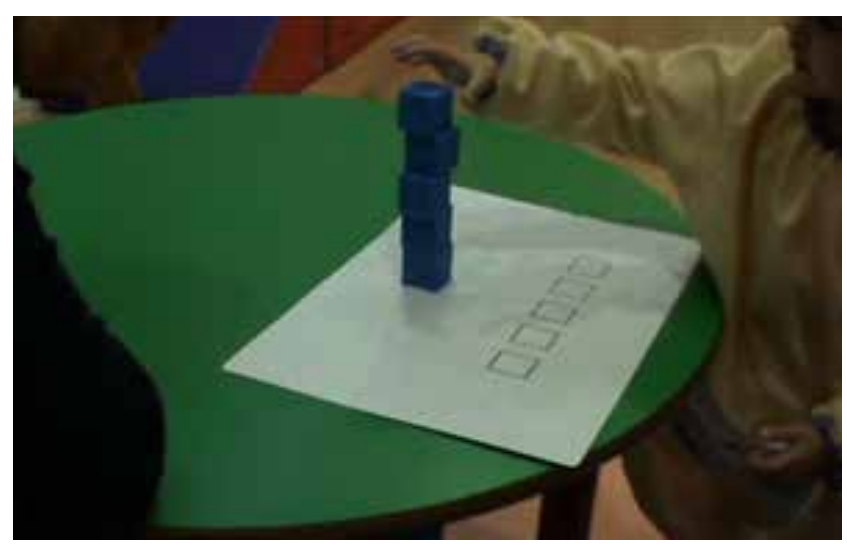

Figura 1: Un niño construyendo la torre de 5 cubos.

La aplicación de la Escala Merrill-Palmer-R, a los niños/as que conformaron la submuestra, se llevó a cabo en los meses de abril y mayo de 2016, en una de las escuelas infantiles situada en Alcalá de Henares (Madrid, España).

\section{Instrumentos y variables}

\section{Los instrumentos empleados para recoger fueron los siguientes:}

Sistema de juguetes inteligentes (torre de cubos): Torre de 5 cubos con sensores incorporados en cada uno de éstos, los cuales permiten recoger información sobre las acciones/movimientos que los niños/as llevan a cabo mientras construyen la torre. Dichos sensores fueron seleccionados de tal manera que tuviesen el tamaño adecuado para ser introducidos dentro de los cubos, que permitiesen recoger información y, finalmente, que tuviesen la capacidad para enviar los datos recogidos al colector (con el fin de ser almacenados) (Figura 2).

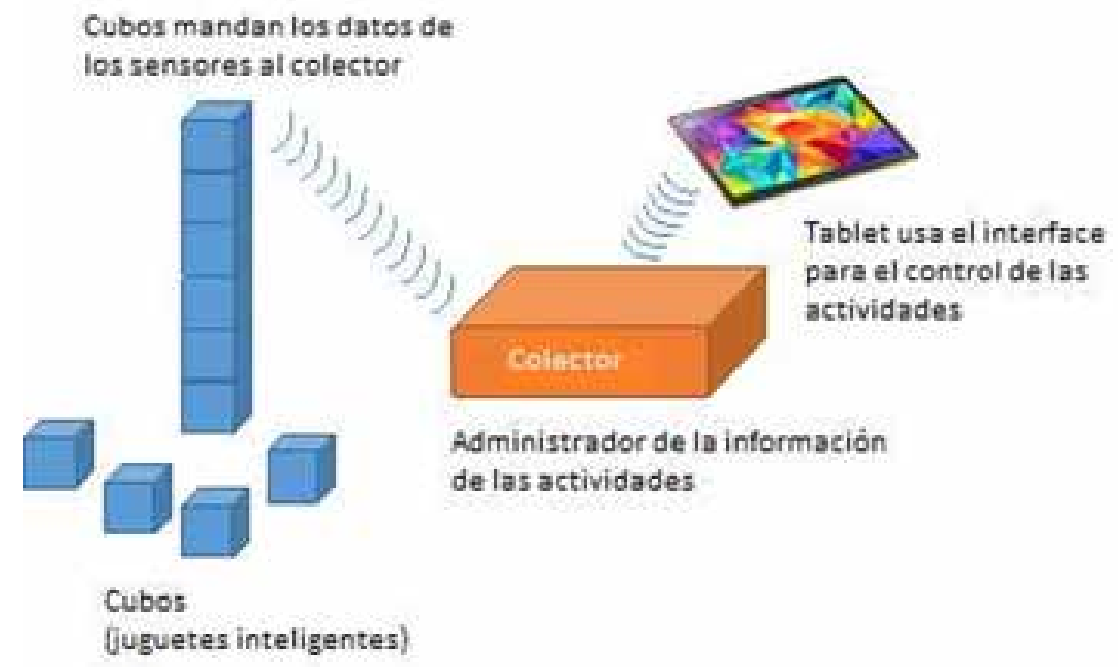

Figura 2. Arquitectura física del funcionamiento del juguete inteligente.

Los cubos envían los datos sensoriales al colector. El colector almacena éstos datos al igual que los datos asociados a la actividad en sí (fecha; hora; responsable de la actividad; datos de identificación del niño/a; etc.). Para una descripción técnica más detallada sobre la arquitectura y componentes del sistema, es posible consultar Rivera, y otros (2016).

Durante la tarea de construcción de la torre, las variables a evaluar mediante este sistema fueron las siguientes: número de cubos que utiliza el/la niño/a para la construcción de la torre (máximo 5); duración o tiempo que tarda en terminar dicha construcción; número de movimientos que realiza el/la niño/a en la ejecución de la tarea; tiempo medio y velocidad media durante el movimiento; velocidad máxima durante el movimiento; momento de velocidad máxima en toda la prueba; mayor velocidad máxima alcanzada por el/la niño/a durante la prueba; menor velocidad máxima; momento de menor velocidad máxima; momento de mayor velocidad máxima, aceleración máxima de movimiento; mayor aceleración máxima; menor aceleración máxima; media de agitaciones (movimientos que hace el/la niño/a que no van dirigidos hacia la construcción de la torre).

Todas estas variables referidas a velocidades, tiempos y agitaciones fueron consideradas en el estudio porque los sensores arrojan datos cuantitativos sobre las mismas. En los resultados se presentarán los datos de las variables que guardan más relación con aspectos del desarrollo de los/as niños/as y con la ejecución eficaz en la tarea de construcción de la torre de cubos. 
Escala de observación: elaborada para determinar el grado de ejecución en latarea de construcción de la torre decubos. Utilizando esta escala, varios jueces calificaron la ejecución en la tarea tras observar la grabación (video) de cada sujeto. Siguiendo los criterios de evaluación, a la ejecución de cada niño/a le fue asignada una puntuación de 0 a 10, valorándola como bien, mal o regular. A continuación, se describen los criterios seguidos para la valoración del grado de ejecución (Tabla 1):

Tabla 1: Criterios para el grado de ejecución.

\begin{tabular}{|c|c|c|}
\hline Valoración & Puntuación & Criterio de evaluación \\
\hline Bien & 10 & $\begin{array}{l}\text { Torre muy alineada al primer intento, en el } \\
\text { espacio indicado para ello, construida a } \\
\text { velocidad alta. } \\
\text { Torre ligeramente desviada al primer intento, } \\
\text { en el espacio indicado para ello, construida a } \\
\text { velocidad media. } \\
\text { Torre muy desviada (pero no se cae) al primer } \\
\text { intento, en el espacio indicado para ello, } \\
\text { construida a velocidad baja y/o con movimientos } \\
\text { intermedios de recolocación. }\end{array}$ \\
\hline Regular & 5 & $\begin{array}{l}\text { Consigue realizar la torre al segundo intento en } \\
\text { el espacio indicado para ello. Consigue realizar } \\
\text { la torre al primer intento en un espacio distinto } \\
\text { al indicado. } \\
\text { Consigue realizar la torre al tercer intento en el } \\
\text { espacio indicado para ello. Consigue realizar la } \\
\text { torre al segundo intento en un espacio distinto } \\
\text { al indicado para ello } \\
\text { Consigue realizar la torre al tercer intento en un } \\
\text { espacio distinto al indicado para ello. }\end{array}$ \\
\hline \multirow[t]{2}{*}{ Mal } & 2 & $\begin{array}{l}\text { Consigue poner cuatro cubos pero al poner el } \\
\text { quinto la torre se cae o no continúa la prueba en } \\
\text { el mejor de los tres intentos. } \\
\text { Consigue poner tres cubos pero al poner el } \\
\text { cuarto la torre se cae o no continúa la prueba en } \\
\text { el mejor de los tres intentos. } \\
\text { Consigue poner dos cubos pero al poner el } \\
\text { tercero la torre se cae o no continúa la prueba } \\
\text { en el mejor de los tres intentos. } \\
\text { Consigue poner un cubo pero al poner el } \\
\text { segundo la torre se cae o no continúa la prueba } \\
\text { en el mejor de los tres intentos. }\end{array}$ \\
\hline & 0 & $\begin{array}{l}\text { No consigue poner ningún cubo a pesar de tener } \\
\text { la intención de hacerlo. }\end{array}$ \\
\hline & 1. $\mathrm{El}$ & ño no tiene intención de construir la torre. \\
\hline $\begin{array}{l}\text { Criterios para } \\
\text { excluir videos: }\end{array}$ & \multicolumn{2}{|c|}{$\begin{array}{l}\text { 2. Otra persona, diferente al sujeto a ser evaluado, toca los } \\
\text { cubos con intención de ayudar a construir la torre (pone algún } \\
\text { cubo en la torre; rearma la torre; evita que la torre caiga). }\end{array}$} \\
\hline
\end{tabular}

Escala de desarrollo Merrill-Palmer Revisada (MP-R) (Roid \& Sampers, 2011): Batería de test de aplicación individual destinada a la evaluación global del desarrollo infantil, validada en población española. Explora específicamente las cinco áreas principales del desarrollo (desarrollo cognitivo; lenguaje y comunicación; desarrollo motor; desarrollo socio-emocional; y conducta adaptativa) en niños de 0 meses a 6 años y medio. Además de estar basada en la evaluación de 'hitos evolutivos', permite detectar cambios pequeños en el funcionamiento del niño/a y evaluar la calidad de su rendimiento. Por último, su uso resulta motivante y estimulante para los niños, incluidos los muy pequeños y otros quienes reciben educación especial. Es de destacar la utilización de juguetes, lo cual hace de este instrumento uno óptimo para la consecución de los objetivos del presente estudio.

\section{Análisis de datos}

Los datos fueron analizados con el paquete estadístico SPSS 23. Primero fueron analizadas las correlaciones entre: el nivel de ejecución en la tarea de construcción de la torre de cubos (observada por los jueces), las medidas recogidas por los sensores integrados en los cubos, y las variables de la Escala Merrill Palmer-R. Acto seguido, fueron analizadas las diferencias entre los niños quienes habían construido la torre bien, regular o mal, específicamente las diferencias en las variables medidas por los sensores y en las áreas del desarrollo evaluadas con la escala Merrill Palmer-R. Análisis no paramétricos fueron realizados debido al desequilibrio del tamaño muestral en los grupos. Por último, las diferencias en función del sexo y de la edad fueron analizadas.

\section{Análisis y resultados}

El desempeño de los/las niños/as en la ejecución de la tarea de construcción de la torre correlaciona con las medidas recogidas por los sensores integrados en los cubos. Concretamente, el grado de ejecución en la construcción de la torre está relacionada de manera positiva con el número de cubos utilizado por el/la niño/a ( $r=.302 ; p<.05)$, y de manera negativa con: el número de movimientos realizados para construir la torre $(r=-.354 ; p<.01)$; la velocidad media durante el movimiento ( $r=-.287$; $p<.05)$; la velocidad máxima durante el movimiento $(r=-.370 ; p<.01)$; la mayor velocidad máxima alcanzada en el movimiento ( $r=-.368 ; p<.01)$; y la mayor aceleración máxima alcanzada en un movimiento ( $r=-.296 ; p<.05)$. Estas relaciones indican que los/ las niños/as que mejor construyen la torre emplean más cubos, hacen menos movimientos y los hacen a menor velocidad y aceleración. Sin embargo, no parece existir relación entre el nivel de ejecución en la construcción de la torre y otras variables (como el tiempo que tarda el niño en construirla (duración)).

El nivel de ejecución en la construcción de la torre está relacionado positivamente con diferentes medidas del desarrollo infantil evaluadas a través de la Escala Merril-Palmer. De éstas, la que muestra correlaciones más altas con el nivel de ejecución es el área de la motricidad gruesa ( $r=.608 ; p<.01)$, seguida del área socioemocional $(r=.483)$, la motricidad fina $(r=.453 ; p<.05)$, el área cognitiva $(r=.416$; $p<.05)$ y el nivel de inteligencia general $(r=.400 ; p<.05)$.

Respecto a las correlaciones entre las variables medidas por los sensores y las áreas del desarrollo infantil evaluadas con la Escala Merrill-Palmer-R, los resultados indicaron que las variables medidas con los sensores y que se relacionan con el grado de ejecución (indicadas anteriormente), también están relacionadas negativamente con alguna de las áreas del desarrollo. Concretamente: el número de movimientos que realiza el/la niño/a para construir la torre está relacionado con la motricidad gruesa ( $r=-.527$; $p<.05)$; la velocidad media durante el movimiento está relacionada con la capacidad de memoria $(r=-.416 ; p<.05)$; la velocidad máxima de movimiento está relacionada con la inteligencia general ( $r=-.456$; $p<.05)$, con el área cognitiva ( $r=-.414 ; p<.05)$, con el lenguaje receptivo $(r=-.419 ; p<.05)$ y con la capacidad de memoria ( $r=-.511 ; p<.01)$; la mayor velocidad máxima alcanzada en el movimiento también está relacionada negativamente con la inteligencia general ( $r=-.419 ; p<.05)$, con el área cognitiva $(r=-.438$; $p<.05)$, con el lenguaje receptivo $(r=-.435 ; p<.05)$ y con la capacidad de memoria $(r=-.459 ; p<.05)$. Además, hay algunas variables que no están relacionadas con el nivel de ejecución en la construcción de la torre, pero sí con algunas de las áreas del desarrollo infantil. De este modo, el tiempo que tarda el/la niño/a en construir la torre (duración) $(r=-.429 ; p<.05)$ y la media de agitaciones de nivel 1 ( $r=-.659 ; p<.01)$ están relacionados con la motricidad gruesa; y el tiempo medio de 
movimientos ( $r=-.455 ; p<.05)$, la media de agitaciones de nivel 3 ( $r=-$ $.417 ; p<.05)$ y la media de agitaciones de nivel $4(r=-.477 ; p<.05)$ están relacionados con el área socioemocional.

Los/las niños/as fueron clasificados en tres grupos en función del grado de ejecución en la construcción de la torre, a saber: mala ejecución, ejecución regular y buena ejecución. Las diferencias entre ellos fueron analizadas, específicamente respecto a las variables medidas con los sensores. Análisis no paramétricos fueron llevados a cabo debido al diferente tamaño muestral de los grupos.

Como es posible observar en la Tabla 2, hay más diferencias significativas entre los grupos extremos, es decir, entre niños/as que construyeron bien la torreyaquellos quienes la construyeron mal. Según la prueba $U$ de Mann-Whitney, los/las niños/as quienes construyeron bien la torre -en comparación con quienes la construyeron malemplearon un número significativamente mayor de cubos $(p=.003)$ y llevaron a cabo menos cantidad de movimientos ( $p=.015)$. Por el contrario, los/las niños/as quienes construyeron mal la torre mostraron una velocidad máxima de movimiento significativamente más alta ( $p=.047)$, mayor velocidad máxima $(p=.004)$, y mayor aceleración máxima ( $p=.019)$. Los/las niños/as quienes construyeron mal la torre se diferencian significativamente de aquellos quienes la construyeron de manera regular, únicamente en la variable mayor velocidad máxima ( $p=.049)$, siendo más elevada los registros de los/las niños/as con mala ejecución. Por último, los/las niños/as quienes construyeron bien la torre se diferencian significativamente de quienes la construyeron regular en cuanto al tiempo medio de movimiento $(p=.006)$, la media de agitaciones de intensidad $2(p=.10)$ y de intensidad 4 ( $p=.004)$, siendo todas ellas mayores en el grupo con buena ejecución.

Tabla 2. Diferencias entre los grupos respecto a la ejecución en la construcción y las variables medidas con los sensores (sólo se muestran las variables en las que existen diferencias significativas entre los grupos)

\begin{tabular}{|c|c|c|c|c|c|c|}
\hline & $\begin{array}{l}\text { Grado de } \\
\text { ejecución en } \\
\text { construcción } \\
\text { de la torre }\end{array}$ & $\mathbf{N}$ & $\begin{array}{c}\text { Rango } \\
\text { promedio }\end{array}$ & $\begin{array}{c}\text { Suma de } \\
\text { rangos }\end{array}$ & $\begin{array}{c}\text { U de Mann- } \\
\text { Whitney }\end{array}$ & $\begin{array}{c}\text { Signific. Asintót. } \\
\text { Bilateral }\end{array}$ \\
\hline \multirow{3}{*}{$\mathrm{N}^{\circ}$ cubos } & $\begin{array}{c}\text { mala } \\
\text { ejecución }\end{array}$ & 6 & 18.33 & 110.00 & \multirow{3}{*}{89.000} & \multirow{3}{*}{.003} \\
\hline & $\begin{array}{c}\text { buena } \\
\text { ejecución }\end{array}$ & 43 & 25.93 & 1115.00 & & \\
\hline & Total & 49 & & & & \\
\hline \multirow{3}{*}{$\begin{array}{c}\mathrm{N}^{\circ} \text { de } \\
\text { movimientos }\end{array}$} & $\begin{array}{c}\text { mala } \\
\text { ejecución }\end{array}$ & 6 & 37.42 & 224.50 & \multirow{3}{*}{54.500} & \multirow{3}{*}{.015} \\
\hline & $\begin{array}{c}\text { buena } \\
\text { ejecución }\end{array}$ & 43 & 23.27 & $100 ., 50$ & & \\
\hline & Total & 49 & & & & \\
\hline \multirow{3}{*}{$\begin{array}{l}\text { Velocidad } \\
\text { máxima de } \\
\text { movimiento }\end{array}$} & $\begin{array}{c}\text { mala } \\
\text { ejecución }\end{array}$ & 6 & 35.83 & 215.00 & \multirow{3}{*}{64.00} & \multirow{3}{*}{.047} \\
\hline & $\begin{array}{c}\text { buena } \\
\text { ejecución }\end{array}$ & 43 & 23.49 & 1010.00 & & \\
\hline & Total & 49 & & & & \\
\hline \multirow{3}{*}{$\begin{array}{l}\text { Mayor } \\
\text { velocidad } \\
\text { máxima }\end{array}$} & $\begin{array}{c}\text { mala } \\
\text { ejecución }\end{array}$ & 6 & 40.83 & 245.00 & \multirow{3}{*}{34.00} & \multirow{3}{*}{.004} \\
\hline & $\begin{array}{l}\text { buena } \\
\text { ejecución }\end{array}$ & 43 & 22.79 & 980.00 & & \\
\hline & Total & 49 & & & & \\
\hline \multirow{3}{*}{$\begin{array}{c}\text { Mayor } \\
\text { aceleración } \\
\text { máxima }\end{array}$} & $\begin{array}{c}\text { mala } \\
\text { ejecución }\end{array}$ & 6 & 37.83 & 227.00 & \multirow{3}{*}{52.000} & \multirow{3}{*}{.019} \\
\hline & $\begin{array}{c}\text { buena } \\
\text { ejecución }\end{array}$ & 43 & 23.21 & 998.00 & & \\
\hline & Total & 49 & & & & \\
\hline \multirow{3}{*}{$\begin{array}{l}\text { Mayor } \\
\text { velocidad } \\
\text { máxima }\end{array}$} & $\begin{array}{c}\text { mala } \\
\text { ejecución }\end{array}$ & 6 & 18.17 & 109.00 & \multirow{3}{*}{26} & \multirow{3}{*}{.049} \\
\hline & $\begin{array}{l}\text { ejecución } \\
\text { regular }\end{array}$ & 19 & 11.37 & 216 & & \\
\hline & Total & 25 & & & & \\
\hline \multirow{3}{*}{$\begin{array}{c}\text { Tiempo } \\
\text { medio } \\
\text { movimiento }\end{array}$} & $\begin{array}{l}\text { ejecución } \\
\text { regular }\end{array}$ & 19 & 22.11 & 420.00 & \multirow{3}{*}{230.000} & \multirow{3}{*}{.006} \\
\hline & $\begin{array}{c}\text { buena } \\
\text { ejecución }\end{array}$ & 43 & 35.65 & 1533.00 & & \\
\hline & Total & 62 & & & & \\
\hline \multirow{3}{*}{$\begin{array}{c}\text { Media de } \\
\text { agitaciones } 2\end{array}$} & $\begin{array}{l}\text { ejecución } \\
\text { regular }\end{array}$ & 19 & 22.63 & 430.00 & \multirow{3}{*}{240.000} & \multirow{3}{*}{.010} \\
\hline & $\begin{array}{c}\text { buena } \\
\text { ejecución }\end{array}$ & 43 & 35.42 & 1523.00 & & \\
\hline & Total & 62 & & & & \\
\hline \multirow{3}{*}{$\begin{array}{c}\text { Media de } \\
\text { agitaciones } 4\end{array}$} & $\begin{array}{l}\text { ejecución } \\
\text { regular }\end{array}$ & 19 & 21.84 & 415.00 & \multirow{3}{*}{225.000} & \multirow{3}{*}{.004} \\
\hline & $\begin{array}{c}\text { buena } \\
\text { ejecución }\end{array}$ & 43 & 35.77 & 1538.00 & & \\
\hline & Total & 62 & & & & \\
\hline
\end{tabular}


Tras analizar las diferencias en las áreas del desarrollo infantil, en función del grado de ejecución en la construcción de la torre (mala, regular y buena), solo es posible observar diferencias estadísticamente significativas en el área de la motricidad gruesa, siendo ésta inferior en los/las niños/as quienes construyeron mal la torre, frente a quienes la construyeron bien ( $p=0.009)$ o de manera regular $(p=0.016)$ (ver Tabla 3). Sin embargo, no existen diferencias significativas en ninguna de las áreas del desarrollo entre los/las niños/as quienes construyeron bien la torre y quienes la construyeron de manera regular.

Tabla 3. Diferencias en las áreas del desarrollo entre los grupos de ejecución de la torre (sólo se muestran las variables en las que existen diferencias significativas)

\begin{tabular}{|c|c|c|c|c|c|c|}
\hline & $\begin{array}{l}\text { Grado de } \\
\text { ejecución en } \\
\text { construcción } \\
\text { de la torre }\end{array}$ & $\mathbf{N}$ & $\begin{array}{c}\text { Rango } \\
\text { promedio }\end{array}$ & $\begin{array}{l}\text { Suma de } \\
\text { rangos }\end{array}$ & $\begin{array}{c}\text { U de Mann- } \\
\text { Whitney }\end{array}$ & $\begin{array}{l}\text { Signific. } \\
\text { Asintót. } \\
\text { Bilateral }\end{array}$ \\
\hline \multirow{3}{*}{$\begin{array}{c}\text { Motricidad } \\
\text { gruesa }\end{array}$} & mala ejecución & 3 & 2.00 & 6.00 & \multirow{3}{*}{.000} & \multirow{3}{*}{.009} \\
\hline & $\begin{array}{c}\text { buena } \\
\text { ejecución }\end{array}$ & 12 & 9.50 & 114.00 & & \\
\hline & Total & 15 & & & & \\
\hline \multirow{3}{*}{$\begin{array}{c}\text { Motricidad } \\
\text { gruesa }\end{array}$} & mala ejecución & 3 & 2.00 & 6.00 & \multirow{3}{*}{.000} & \multirow{3}{*}{.016} \\
\hline & $\begin{array}{l}\text { ejecución } \\
\text { regular }\end{array}$ & 7 & 7.00 & 49.00 & & \\
\hline & Total & 10 & & & & \\
\hline
\end{tabular}

Respecto a las diferencias en el desarrollo infantil, en función del género (Tabla 4), únicamente se encuentran diferencias significativas entre niños y niñas en el área de conducta adaptativa y autocuidado
( $p=.027)$, siendo mayor en las niñas. Es preciso anotar que los niños y niñas no difieren significativamente en ninguna de las variables medidas a través de los sensores en los cubos.

Tabla 4. Diferencias en las áreas del desarrollo entre niños y niñas (sólo se muestran las variables en las que existen diferencias significativas)

\begin{tabular}{ccccccc} 
& $\begin{array}{c}\text { Género del } \\
\text { sujeto }\end{array}$ & N & $\begin{array}{c}\text { Rango } \\
\text { promedio }\end{array}$ & $\begin{array}{c}\text { Suma de } \\
\text { rangos }\end{array}$ & $\begin{array}{c}\text { U de Mann- } \\
\text { Whitney }\end{array}$ & $\begin{array}{c}\text { Signific. } \\
\text { Asintót. } \\
\text { Bilateral }\end{array}$ \\
$\begin{array}{c}\text { Conducta } \\
\text { adaptativa }\end{array}$ & Hombre & 13 & 9.88 & 128.50 & & \\
$\begin{array}{c}\text { y } \\
\text { autocuidad }\end{array}$ & Mujer & 12 & 16.38 & 196.50 & 37.500 & .027 \\
\hline
\end{tabular}

Para analizar los datos en función de la edad (ver Tabla 5), se crearon cuatro grupos con los siguientes rangos de edad: 24-30 meses; 30-36 meses; 36-42 meses; y 42-48 meses (éste último fue eliminado por estar integrado solamente por un niño). En función de la edad, únicamente difieren significativamente en la variable duración, lo que indica que los/las niños/as mayores (36-42 meses) tardan menos realizando la tarea, en comparación con aquellos en otros grupos de edad. También es posible observar que la diferencia en el número de movimientos está muy próxima a ser significativa.

Tabla 5. Diferencias en las áreas del desarrollo en función de la edad (sólo se muestran las variables en las que existen diferencias significativas)

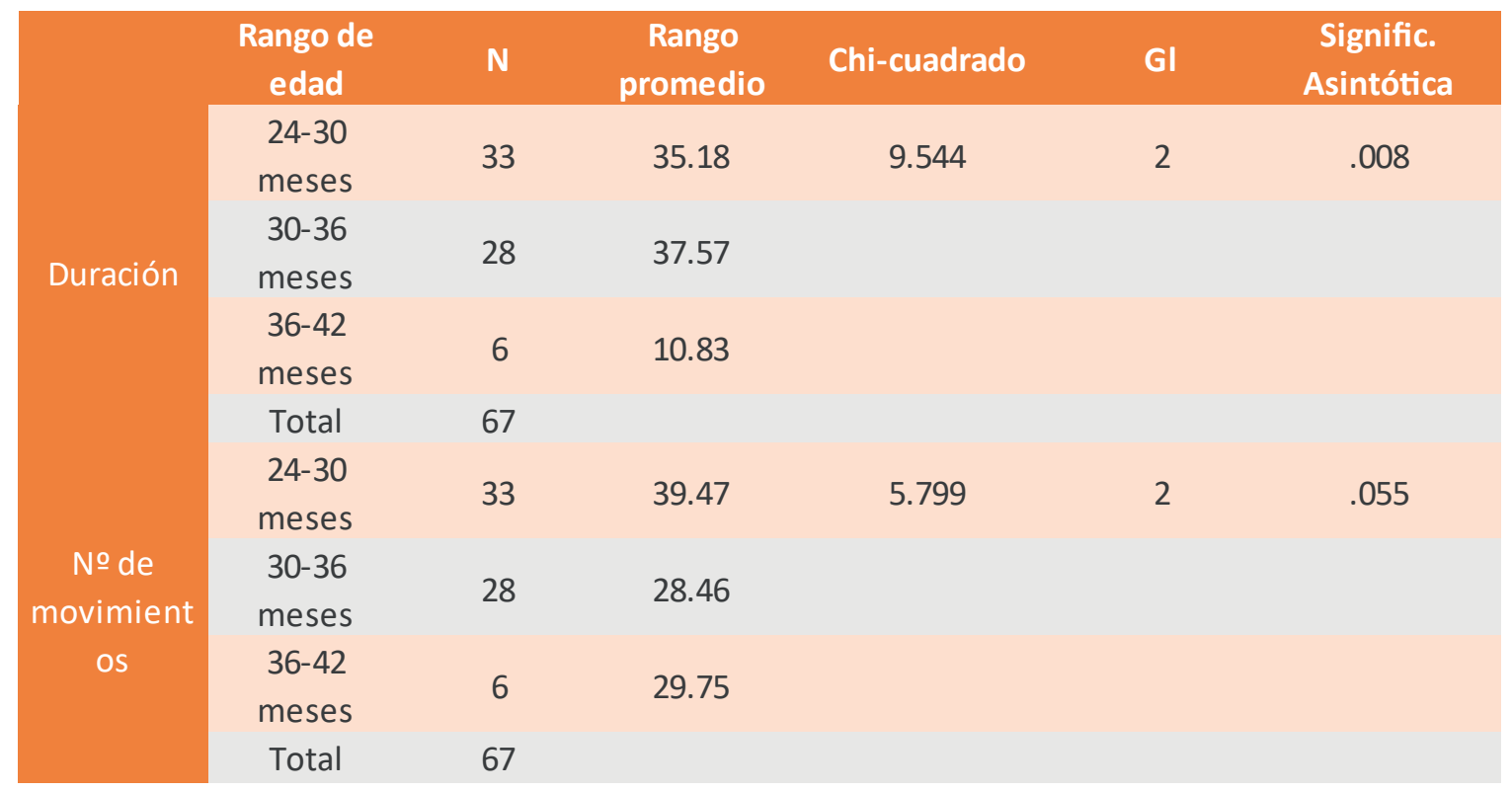




\section{Discusión y conclusiones}

Las relaciones manifiestas permiten concluir que las variables más relevantes en la construcción apropiada de la torre son el número de cubos; el número de movimientos; y la velocidad y aceleración de los movimientos que los/las niños/as realizan. Lo anterior indica que los niños y niñas que mejor construyen la torre emplean los cinco cubos, hacen menos movimientos con menor velocidad y aceleración, que aquellos quienes la construyen mal. En función del género, los niños y niñas no difieren significativamente en ninguna de las variables medidas con los sensores en los cubos. Sin embargo, sí hay diferencias en función de la edad, en tanto los niños y niñas de mayor edad construyen la torre en menos tiempo que los más pequeños.

Un aspecto a destacar es que no todas las variables sirven para diferenciar entre los distintos grados de ejecución en la construcción de la torre. Aquellas que sí permiten diferenciarlos son: la mayor velocidad máxima (que permite diferenciar entre una ejecución mala y una regular) y el tiempo medio de movimiento y la media de agitaciones (que permiten distinguir más entre una ejecución buena y una regular). Lo anterior lleva a concluir que ampliar la muestra es una necesidad para poder añadir otras variables que permitan diferenciar con más detalle estos grados de ejecución en la tarea, y que nutran los datos que recogen los sensores del juguete inteligente. Además, éstas son variables que recién empiezan a ser estudiadas y, por consecuente, han sido poco comparadas con otros estudios publicados.

De los datos recabados por los cubos inteligentes, las medidas que se relacionan con más áreas del desarrollo infantil (evaluadas a través de la Escala Merril Palmer-R) son: la velocidad máxima de movimiento (que indica la capacidad para realizar un movimiento en el mínimo tiempo) y la mayor velocidad máxima alcanzada por el/la niño/a durante la prueba.

Un hallazgo a destacar es la relación positiva entre el nivel de ejecución en la construcción de la torre y medidas del desarrollo infantil como la motricidad gruesa, con la que presenta las correlaciones más altas. De todas las variables del desarrollo infantil evaluadas ésta parece ser la más relevante, dado que en función de la motricidad gruesa es posible diferenciar más los/las niños/as que construyen la torre bien y/o de manera regular de aquellos quienes la construyen mal. Respecto a las otras medidas del desarrollo, y en función de su correlación con nivel de ejecución, al área motriz viene seguida por el área socioemocional; la motricidad fina, área cognitiva; y nivel de inteligencia general.

El hecho que algunas de las variables evaluadas mediante los cubos se relacionen con distintas áreas del desarrollo y, sobre todo, con la motricidad gruesa, hace pensar que vamos en buena dirección a la hora de plantearnos que dichas variables pueden ayudar en la detección precoz de problemas en los niños. De hecho, estos hallazgos reiteran el importante papel de la evaluación de aspectos motores (Roid \& Sampers, 2011) y confirman su relación con problemas o dificultades en el desarrollo (Alcantud, Alonso, \& Rico, 2015; Roid \& Sampers, 2011; Iceta \& Yoldi, 2002).

No en vano existen investigaciones previas que relacionan la motricidad con diferentes trastornos del desarrollo. Por ejemplo, estudios centrados en los trastornos del espectro autista han revelado diferencias significativas en el desarrollo de habilidades motrices finas y gruesas en niños con trastorno de Asperger (Provost \& Heimerl, 2007; Lloyd, MacDonald, \& Lord, 2013). Respecto a los/las niños/as quienes presentan alguna alteración sensorial, Webber, Wood, Gole, \& Brown (2008) encontraron que los niños con ambliopía (en particular aquellos con estrabismo) presentaban déficits en el rendimiento motor en tareas de destreza manual que requerían velocidad y precisión, en comparación con los sujetos del grupo control. También se han evidenciado destrezas motrices más pobres en niños con Trastorno por Déficit de Atención e Hiperactividad (TDAH) (Pieck, J.; Pitcher, T.; Hay, D., 1999); peores resultados en los subtests de destreza manual por parte niños mayores (peor calidad en la escritura y mayor rapidez en el dibujo); resultados fácilmente explicables por las características del trastorno (Flapper, Houwen, \& Schoemaker, 2006).

Investigaciones previas como las citadas anteriormente (Provost \& Heimerl, 2007; Lloyd, MacDonald, \& Lord, 2013; Webber, Wood, Gole, \& Brown, 2008; Pieck, J.; Pitcher, T.; Hay, D., 1999) confirman la relación entre la motricidad y diferentes trastornos del desarrollo. Sin embargo, y de cara a futuras investigaciones, será necesario estudiar a fondo si existe relación entre las medidas que aportan los cubos y las acciones/ movimientos de niños/as con un diagnóstico concreto. Además de la necesidad ya mencionada de ampliar la muestra para poder comparar a niños/as con y sin problemas de desarrollo.

En general, los resultados de este estudio apoyan la adecuación de los cubos inteligentes como instrumento de evaluación y, además, sugieren que sirven para orientar futuras investigaciones que permitan validar, de manera consistente, su utilidad para la detección de problemas del desarrollo. De ser así, este juguete inteligente contribuiría al desarrollo de medidas de evaluación del movimiento de niños/ as, más precisas y directas que las desarrolladas en otros trabajos (Albinali, Goodwin, \& Intille, 2012), (con sensores corporales) o (SmitsEngelsman, Wilson, Westenberg, \& Duysens, 2003), (con lápiz digital)).

En definitiva, el aporte principal de este estudio exploratorio es la evidencia sobre la existencia de relaciones entre distintas variables que pueden medirse con los cubos inteligentes, el grado de ejecución en la tarea y distintas variables del desarrollo.

Consideramos que hay un horizonte abierto en el que los avances tecnológicos y la detección precoz de problemas del desarrollo en niños/as se encuentran, y que ésta conjunción nos acerca a una atención más temprana posible de los mismos. Por el momento, estamos en el proceso de crear otros juguetes inteligentes (concretamente sonajero, espigas y pelota) que sirvan este mismo fin para niños y niñas de otras edades.

\section{Referencias}

Albinali, F.; Goodwin, M.S. e Intille, S.(2012). Detecting stereotypical motor movements in the classroom using accelerometry and pattern recognition algorithms.PervasiveMob. Comput. 8, 103-114. Recuperado https://www. sciencedirect.com/science/article/pii/S1574119211000423?via\%3Dihub

Alcantud, F.; Alonso, Y.; Rico, D. (2015) Sistema de Detección Precoz de Trastornos del Desarrollo (SDPTD). Real Patronato sobre Discapacidad. Ministerio de Sanidad, Servicios Sociales e Igualdad. Recuperado de http://roderic.uv.es/ handle/10550/58855

Álvarez Gómez, M.; Soria, J.; Galbe, J. (2009) Importancia de la vigilancia del desarrollo psicomotor por el pediatra de atención primaria: revisión del tema y experiencias de seguimiento en una consulta en Navarra. Revista de Pediatría en Atención Primaria, 11(41), 65-87. Recuperado de http:// scielo.isciii.es/scielo.php?pid=S1139-76322009000100005\&script=sci arttext\&tlng=en

Ashton, K. (2009). That Internet of ThingsThing. RFID Journal. Recuperado de http://www.rfidjournal.com/article/print/4986 
Barrera, L., Perdomo, B., Serrato, M., Trujillo, Y. (2014) Impacto de los juguetes en los procesos formativos de los niños y niñas. (Trabajo de grado, Universidad de Manizales). Recuperado de http://ridum.umanizales.edu.co:8080/ xmlui/bitstream/handle/6789/1965/Perdomo Ordo\%C3\%Blez Bleyi Constanza_2014.pdf? sequence $=1 \& i s$ Allowed $=y$

Boss, S., Bruce, H., Charlie, C; Miller, K. (2001). "Developing Smart Toys--From Idea To Product". Intel Technology Journal. Retrieved 2007-11-03. Recuperado de https://www.intel.com/content/dam/www/public/us/en/documents/ research/2001-vol05-iss-4-intel-technology-journal.pdf

Brown, R. (2000) Social identity theory: Past achievements, current problems and future challenges. European Journal of Social Psychology, 30(6), 745-778. Recuperado https://onlinelibrary.wiley.com/doi/abs/10.1002/10990992(200011/12)30:6\%3C745::AID-EJSP24\%3E3.0.CO;2-O

Brunet, O. y Lezine, I. (1980). El desarrollo psicológico en la primera infancia. Madrid: Pablo del Rio. Recuperado de https://www.iberlibro.com/buscarlibro/titulo/desarrollo-psicologico-primera-infancia-manual-seguimiento/ autor/brunet-i-lezine/

Canal, R., García, P., Touriño, E., Santos, J., Martín, V., Ferrari, M. J., Martínez, M., Guisuraga, Z., Boad, L., Rey, F., Franco, M. y Fuentes, J. (2006). La detección precoz del autismo. Intervención psicosocial, 15(1), 29-47. Recuperado de http://scielo.isciii.es/pdf/inter/v15n1/v15n1a03.pdf

Costa, M.; Romero, M.; Mallebrera, C.; Fabregat, M.; Torres, E.; Martínez, MJ.; Martínez, Y.; Zaragoza, R.; Torres, S.; Martínez, P. (2007) Juego, juguetes y discapacidad: La importancia del Diseño Universal. AIJU. Ibi (Alicante). Recuperado de http://www.cesya.es/sites/default/files/documentos/ folleto\%20AIJU.pdf

Costa, M.; Torres, E.; Romero, M. T.; Fabregat, M.; Torres, E.; Martínez, Y.; Mallebrera, C.; Martínez, M.J.; Zaragoza, R.: Martínez, P.; Millá, M.G.; Crespo, J.; Gabaldón, M.C.; Richart, E.; Munera, L.; Hernández, E.M. y García, J (2008) Juego, juguetes y atención temprana. Pautas para el diseño de juguetes útiles en la terapia psicopedagógica. AIJU. Ibi (Alicante). Recuperado de http://www.caib.es/sacmicrofront/archivopub. do?ctrl=MCRST2745ZI115002\&id=115002

Brazelton, T. Nugent, K. \& Costas, C. (1997). Escala para la evaluación del comportamiento neonatal. Barcelona, España: Paidos Iberica. Recuperado de https://www.agapea.com/libros/Escala-para-la-evaluacion-delcomportamiento-neonatal-9788449304293-i.htm\#valorarLibro

Cools, W.; Martelaer, K.; Samaey, C. y Andries C. (2008). Movement skill assessment of typically developing preschool children: A review of seven movement skill assessment tools. Journal of SportsScience and Medicine 8, 154-168. Recuperado de https://www.ncbi.nlm.nih.gov/pmc/articles/ PMC3761481/

De Miguel, T. (2001). La Internet del futuro y la I+D. LYCHNOS, Cuadernos de la Fundación General CSIS, 7, 55-60. Recuperado de http://www.fgcsic.es/ lychnos/es es/articulos/la internet del futuro y la id

Diario de Alcalá (2016, 30 de octubre) La UAH 'fabrica' juguetes inteligentes para la detección precoz de problemas en el desarrollo.

EDUCERE (2013) https://educeremus.wordpress.com/

EIDD (2004). La Declaración de Estocolmo del EIDD. Estocolmo: European Institute for Design and Disability. Recuperado http://dfaeurope.eu/what-is-dfa/dfadocuments/the-eidd-stockholm-declaration-2004/

Flapper, B. C.T.; Houwen, S. y Schoemaker, M.M. (2006). Fine motor skills and effects of methylphenidate in children with attention-deficit-hyperactivity disorder and developmental coordination disorder. Developmental Medicine and ChildNeurology, 48 (3), 165-169. Recuperado de https://www. cambridge.org/core/journals/developmental-medicine-and-child-neurology/ article/fine-motor-skills-and-effects-of-methylphenidate-in-children-withattentiondeficithyperactivity-disorder-and-developmental-coordinationdisorder/FBD6CF347709A9AB39A72F8396D85F7C

García Sánchez, F.A.; Mendieta, P. y Castellanos, P. (2010). Evaluación del niño en atención temprana. Universidad de Murcia y ASTRAPACE. Recuperado de https://evaluacionatenciontemprana.wordpress.com/2012/12/25/evaluaciondel-nino-en-atencion-temprana/

Iceta, A. y Yoldi, M.E. (2002) Desarrollo psicomotor del niño y su valoración en atención primaria. ANALES Sis San Navarra, Vol. 25 (Suplemento 2), 35-43. Recuperado de https://www.santafe.gob.ar/index.php/educacion/ content/download/149387/732092/file/Desarrollo\%20psicomotor\%20del\%20 ni\%C3\%B10.pdf

Jelinek, L., Peters, G., Okuley, J., McGowan, S. (2001). Dissection of the Intel Play QX3 Computer Microscope. Intel Technology Journal, 4, 1-10. Recuperado de https://www.intel.com/content/dam/www/public/us/en/documents/ research/2001-vol05-iss-4-intel-technology-journal.pdf
Josse, D. (2001). Brunet-LézineRévisé: Échelle de développementpsychomoteur de la petite enfance. París: Établissementsd'ApplicationsPsychotechniques. Recuperado de https://www.ecpa.fr/psychologie-clinique/test.asp?id=1416

Josse, D. (1997). Brunet-LézineRevisado. Escala de Desarrollo Psicomotor de la Primera Infancia. Madrid: Psymtéc. Recuperado de http://vufind.uniovi.es/ Record/1215909/Details\#tabnav

Lipp, P. y Frekel, L.A. (2000). Engineers and computer scientists are turning their passion for play into exciting careers at toy companies. Graduate Engineer \& Computer Careers, Magazine, 1-5. Recuperado de http://www. graduatingengineer.com/articles/20000728/Fun-and-Games

Lloyd, M.; MacDonald, M. y Lord, C. (2013). Motor skills of toddlers with autism spectrum disorders. Autism, 17(2), 133-146.doi: https://doi. org/10.1177/1362361311402230

Manga, D. y. Ramos, F. (2001). Evaluación de los síndromes neuropsicológicos infantiles. Revista de Neuropsicología, 32 (7), 664-675. Recuperado de https://www.researchgate.net/profile/Francisco_Camposil/ publication/11946936 Evaluation of children's neuropsychological syndromes/links/57910a3208ae4e917d0469f7.pdf

McCarthy, D. (1996). MSCA. Escalas McCarthy de Aptitudes y Psicomotricidad para niños. Madrid: TEA Ediciones. Recuperado de https://www.amazon.es/ MSCA-Escalas-McCarthy-Aptitudes-Psicomotricidad/dp/8471748487

Martin-Ruiz, M.L., Valero, M.A., Linden M., Nunez-Nagy, S. y Gutierrez, A. (2015) Foundations of a smart toy development for the early detection of motoric impairments at childhood. International Journal of Pediatric Research, 1 (2), 1-3. doi: https://doi.org/10.23937/2469-5769/1510011

Newborg, J.; Stock, J. y Wnek, L. (1998). Inventario de Desarrollo Battelle. Adaptación Española. Madrid: TEA Ediciones. Recuperado de https://www.casadellibro.com/libro-battelle-inventario-de-desarrol lo/9788471749826/1893743

Nuñez-Nagy,S.; Alarcos, B.; Valero, M.A., Gallego, T.; Ramírez, R.J.; Serrano, C. (2015) Smart toys for detecting children developmental delays. Poster presentado a la International Conference on Recent advances in Neurorehabilitation. Valencia, España. doi: https://doi. org/10.3390/s16111953. Recuperado de https://www.researchgate.net/ publication/282610945 Smart toys for detecting children developmental delays

Organization Mundial de la Salud. (2011). World report on disability. Geneva, Avenue Appia. Recuperado de http://www.who.int/disabilities/world report/2011/report.pdf

Pieck, J.P.; Pitcher, T.M. y Hay, D.A. (1999). Motor coordination and kinesthesis in boys with attention deficit-hyperactivity disorder. Developmental Medicine and ChildNeurology, 41 (3), 159-165. Recuperado de https://www. cambridge.org/core/journals/developmental-medicine-and-child-neurology/ article/motor-coordination-and-kinaesthesis-in-boys-with-attentiondeficithyperactivity-disorder/E24D96B12AF7F4DC472C821CFF63CBE0\#fnd tn-metrics

Plowman, L. y Luckin, R. (2004) Interactivity, Interfaces, and Smart Toys. Computer, 37 (2). pp. 98-100. Recuperado de https://ieeexplore.ieee.org/ abstract/document/1266302/

Provost, B.; Heimerl, S. y López, B.R. (2007). Levels of Gross and Fine Motor Development in Young Children with Autism Spectrum Disorder. Physical and Occupational Therapy in Pediatrics, 27 (3), 21-36. Recuperado de https://www.tandfonline.com/doi/abs/10.1080/J006v27n03 03

Provost, B.; Lopez, B.R. y Heimerl, S. (2006). A comparison of motor delays in young children: Autism spectrum disorder, developmental delay, and developmental concerns. Journal of Autism and Developmental Disorders, 37, 321-328. Recuperado de https://link.springer.com/article/10.1007/s10803006-0170-6

Ramus, F.; Pidgeon, E. y Frith, U. (2003) The relationship between motor control and phonology in dyslexic children. Journal of Child Psychology and Psychiatry, 44(5), 712-722. Recuperado de https://onlinelibrary.wiley.com/ doi/abs/10.1111/1469-7610.00157

Rivera, D.; García, A.; Alarcos, B.; Velasco, J.R.; Ortega, J.E. y Martínez-Yelmo, I. (2016). Smart Toys Designed for Detecting Developmental Delays. Sensors 16 (11).doi: https://doi.org/10.3390/s16111953. Recuperado de http://www. mdpi.com/1424-8220/16/11/1953

Robaina, G.R.; Riesgo, S de la C; Robaina, M.S. (2007). Evaluación Diagnóstica del niño con Parálisis Cerebral. Revista Cubana de Pediatría, 79 (2) 1-13. Recuperado de http://scielo.sld.cu/scielo.php?pid=S0034$75312007000200007 \&$ script $=s c i$ arttext\&tlng $=p t$

Rodríguez-Porrero, C.; Gil González, S (2015). Reto 7. Diseño para todos en educación. Madrid. CEAPAT. Recuperado de http:/www ceapat.es/ 
InterPresent2/groups/imserso/documents/binario/reto educ.pdf

Roid, G.H. ySampers, J. L. (2011) Escalas de desarrollo Merrill-Palmer-R. Adaptación española. TEA Ediciones. Recuperado de http://www.web. teaediciones.com/Ejemplos/Extracto Manual MPR web.pdf

Ruiz, L., Linaza, J., Peñaloza, R. (2008). El estudio del desarrollo motor: entre la tradición y el futuro. Revista Fuentes, 8 (1), 1-16. Retrieved from: https:// revistascientificas.us.es/index.php/fuentes/article/view/2527/2387

Secadas, F. (2009) (3edición). Escala Observacional de Desarrollo. Madrid, TEA. Recuperado de https://www.casadellibro.com/libro-eod-escalaobservacional-del-desarrollo/9788471749550/1893733

Serrano, C. y Laorden, C. (2015) Juguetes inteligentes como apoyo a la detección de trastornos motóricos en educación temprana. XII Congreso de Educación Especial, marzo. Universidad Complutense de Madrid.

Shwe, Helen (1999). Smarter Play for Smart Toys: The Benefits of TechnologyEnhanced Play. ZowieIntertainment, Inc. Retrieved 2007-11-03. Recuperado de https://zowie.benq.com/en/support.html?searchWord=Smarter\%2520Play \%2520for\%2520Smart\%2520Toys:\%2520The\%2520Benefits\%2520of\%2520Tec hnology-Enhanced\%2520Play\&\#SearchBar

Smits-Engelsman, B.C.M.; Wilson, P.H.; Westenberg, Y. Y Duysens, J. (2003). Fine motor deficiencies in children with developmental coordination disorders and learning disabilities: an underlying open-loop control deficit. Human Movement Science, 22, (4-5) 495-513. Recuperado de https://www. sciencedirect.com/science/article/abs/pii/S016794570300068X

Tseng, M. H., Henderson, A., Chow, S. y Yao, G. (2004). Relationship between motor proficiency, attention, impulse, and activity in children with ADHD. Developmental Medicine y ChildNeurology, 46(6), 381-388. Recuperado de https:/www.cambridge.org/core/journals/developmental-medicine-andchild-neurology/article/relationship-between-motor-proficiency-attentionimpulse-and-activity-in-children-with-adhd/44665B66135A1E1A3C7458310B C9B82D

Valero, M.A. (2001). Tecnologías para la educación inclusiva: de la integración a la interacción. En Arnaiz, P., Hurtado, Ma .D. y Soto, F.J. (Coords.) (2010). 25 Años de Integración Escolar en España: Tecnología e Inclusión en el ámbito educativo, laboral y comunitario. Murcia: Consejería de Educación, Formación y Empleo. Recuperado de https://www. researchgate.net/ publication/246726690_Tecnologias_para_la_educacion_inclusiva_de_la_ integracion a la interaccion.

Vericat, A. y Orden, B.A. (2013) (2013) El desarrollo psicomotor y sus alteraciones: entre lo normal y lo patológico. Ciência y SaúdeColetiva, 18(10): 29772984. Recuperado de https://www.scielosp.org/scielo.php?pid=S1413$81232013001800022 \&$ script $=$ sci arttext\&tlng $=e n$

Webber, A.L.; Wood, S.M.; Gole, G.A. y Brown, B. (2008). The effect of Amblyopia on Fine Motor Skills in Children. Investigative Ophtahalmology and Visual Science, 49(2), 594-603. Recuperado de https://iovs. arvojournals.org/article. aspx?articleid $=2164353$ 
Aproximación a la validación de un juguete inteligente como apoyo en la detección precoz de problemas del desarrollo 